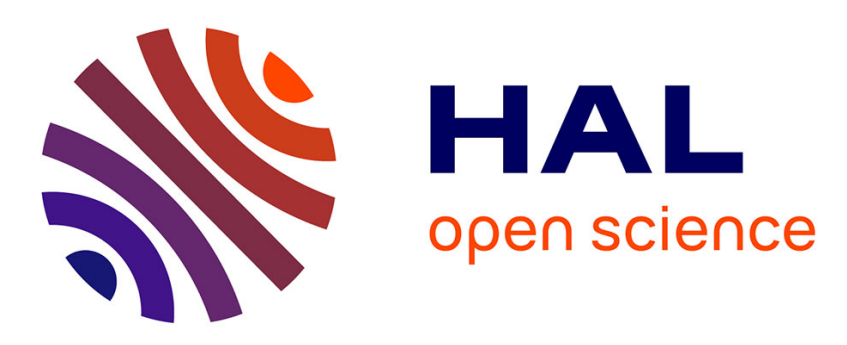

\title{
Multiple Scattering Effects in the Interplanetary Medium: Evaluation Using SOHO SWAN and MAVEN EUVM Lyman $\alpha$ Measurements
}

Eric Quémerais, Edward Thiemann, Martin Snow, Stéphane Ferron, Walter Schmidt

\section{To cite this version:}

Eric Quémerais, Edward Thiemann, Martin Snow, Stéphane Ferron, Walter Schmidt. Multiple Scattering Effects in the Interplanetary Medium: Evaluation Using SOHO SWAN and MAVEN EUVM Lyman $\alpha$ Measurements. Journal of Geophysical Research Space Physics, 2019, 124 (6), pp.3949-3960. 10.1029/2019JA026674 . insu-02151127

\section{HAL Id: insu-02151127 https://hal-insu.archives-ouvertes.fr/insu-02151127}

Submitted on 11 Sep 2019

HAL is a multi-disciplinary open access archive for the deposit and dissemination of scientific research documents, whether they are published or not. The documents may come from teaching and research institutions in France or abroad, or from public or private research centers.
L'archive ouverte pluridisciplinaire HAL, est destinée au dépôt et à la diffusion de documents scientifiques de niveau recherche, publiés ou non, émanant des établissements d'enseignement et de recherche français ou étrangers, des laboratoires publics ou privés. 


\section{JGR Space Physics}

\author{
RESEARCH ARTICLE \\ 10.1029/2019JA026674 \\ Key Points: \\ - We present a new method to evaluate \\ the solar flux toward any object in the \\ solar system \\ - We evaluate the contribution of \\ multiple scattering to the \\ interplanetary ultraviolet emission
}

Correspondence to:

E. Quémerais,

eric.quemerais@latmos.ipsl.fr

Citation:

Quemerais, E., Thiemann, E. M. B., Snow, M., Ferron, S., \& Schmidt, W. (2019). Multiple scattering effects in the interplanetary medium: Evaluation using SOHO SWAN and MAVEN EUVM Lyman $\alpha$ measurements. Journal of Geophysical Research: Space Physics, 124, 3949-3960. https://doi.org/10.1029/ 2019JA026674

Received 1 MAR 2019 Accepted 16 MAY 2019 Accepted article online 23 MAY 2019 Published online 20 JUN 2019
(C)2019. The Authors.

This is an open access article under the terms of the Creative Commons Attribution-NonCommercial-NoDerivs License, which permits use and distribution in any medium, provided the original work is properly cited, the use is non-commercial and no modifications or adaptations are made.

\section{Multiple Scattering Effects in the Interplanetary Medium: Evaluation Using SOHO SWAN and MAVEN EUVM Lyman $\alpha$ Measurements}

\author{
Eric Quémerais ${ }^{1}$ (D), Edward Thiemann ${ }^{2}$ iD, Martin Snow $^{2}$ iD, Stéphane Ferron ${ }^{3}$, \\ and Walter Schmidt ${ }^{4}$ iD
}

${ }^{1}$ LATMOS-OVSQ, Université Versailles Saint-Quentin, Guyancourt, France, ${ }^{2}$ Laboratory for Atmospheric and Space Physics, University of Colorado Boulder, Boulder, CO, USA, ${ }^{3}$ ACRI-ST, Guyancourt, France, ${ }^{4}$ Finnish Meteorological Institute, Helsinki, Finland

\begin{abstract}
Interplanetary background measurements can be used to derive the solar flux that is illuminating the atoms in the interplanetary medium. We present a new algorithm combining measurements from the Solar Wind ANisotropies (SWAN)-Solar Heliospheric Observatory (SOHO) Lyman $\alpha$ photometer with Earth-based solar flux measurements to derive the solar illuminating flux at Lyman $\alpha$ in any direction along the ecliptic plane. The modulations of the solar flux and the interplanetary background intensity are proportional. The proportionality coefficient is a direct measurement of the fraction of the interplanetary brightness that is due to single scattering in the interplanetary medium. We find that for an observer at $1 \mathrm{AU}$ from the Sun, single scattering photons represent roughly $70 \%$ of the total brightness. This new algorithm is tested by a comparison with actual measurements from the Extreme Ultraviolet Monitor (EUVM)-Mars Atmosphere and Volatile EvolutioN (MAVEN) solar monitor in orbit around Mars since 2014.
\end{abstract}

\section{Introduction}

The heliosphere is filled with neutral atoms coming from the interstellar medium, mainly hydrogen and helium. Because of the relative motion between the solar system and the local interstellar cloud, these neutral atoms can reach the inner heliosphere before they are ionized by charge exchange with solar wind protons or photoionized by extreme ultraviolet (EUV) photons. The direction from which the interstellar atoms are coming is called upwind. The upwind direction for hydrogen atoms has been determined by hydrogen absorption cell measurements (Quémerais et al., 1999). It is defined as $252.3 \pm 0.7^{\circ}$ in ecliptic longitude and $8.7 \pm 0.9^{\circ}$ in ecliptic latitude. Downwind is the opposite direction in the sky. In the inner heliosphere, the neutral atoms are mainly ionized by charge exchange with solar wind protons or by photoionization. The remaining neutrals resonantly scatter the solar photons. This emission is called the interplanetary ultraviolet background. Its main component corresponds to resonant scattering of the solar H Lyman $\alpha$ photons $(121.56 \mathrm{~nm})$.

The interplanetary background at Lyman $\alpha$ has been studied for almost 50 years. Since the first measurements by Bertaux and Blamont (1971) and Thomas and Krassa (1971), several instruments have obtained data of this emission due to the backscattering of the solar Lyman $\alpha$ photons by hydrogen atoms in the interplanetary medium (Ajello et al., 1987; Quémerais et al., 2013). The largest data set of interplanetary background measurements, spanning more than two decades, has been obtained by the Solar Wind ANisotropies (SWAN) instrument aboard the Solar Heliospheric Observatory (SOHO) spacecraft (Bertaux et al., 1995). Although it was not expected originally, it was found that the study of the SWAN data could be used to infer the spatial and temporal variations of the solar illuminating flux at Lyman $\alpha$. Quémerais and Bertaux (2002) implemented an algorithm that allowed one to derive the flux emitted by the Sun at any given moment and in any direction based on a linear correlation obtained between the ratio of SWAN background measurements with the variations of the solar flux as monitored from the Earth.

One application of this algorithm was to estimate the solar Lyman $\alpha$ irradiance at any planet in the solar system at any time. This method complements the time-shifting method which is usually used to evaluate 
the flux changes between the irradiance measured at Earth and the flux illuminating a planet in a different part of the solar system. The time-shifting method neglects temporal variations of the solar flux and simply rotates the Sun to find when the area of the Sun illuminating the planet at the time of observation was last aligned with Earth. It can be improved by finding the next measurement when the same region is aligned with Earth and by using a linear interpolation between the two flux values (see Thiemann et al., 2017). However, due to the lack of actual measurements of the solar flux made at a planet, it was previously not possible to evaluate if the SWAN method is really more efficient than the time-shifting method.

The Mars Atmosphere and Volatile EvolutioN (MAVEN; Jakosky et al., 2015) mission orbiting Mars since 2014 allows us to finally answer this question. MAVEN includes the Extreme Ultraviolet Monitor (EUVM, Eparvier et al., 2015), which measures calibrated solar Lyman $\alpha$ irradiance at Mars. This is the first time that an instrument monitoring the solar input has obtained data over a long period of time while in orbit around a planet other than the Earth, in this case Mars. The measurements of EUVM-MAVEN give us the opportunity to validate the method of estimating the solar irradiance in a given direction using SWAN data.

Section 2 will introduce a general method to derive the solar flux from the interplanetary background data. This is an improvement over the original method presented in Quémerais and Bertaux (2002). The new method allows us to express the relation between the modulations of the solar flux and the interplanetary background as a function of single and multiple scattering terms in the interplanetary medium. In section 3, we will apply this relation to the comparison of the EUVM-MAVEN and SWAN-SOHO data sets in order to determine the relevant parameters. Finally, we will apply the new method to estimate the solar Lyman $\alpha$ flux toward Mars from the SWAN data and compare the results to the EUVM measurements and to the time-shifting method.

\section{Retrieving Solar Flux From the Interplanetary Background}

\subsection{Modulations of the Interplanetary Background Data}

The hydrogen atoms present in the interplanetary medium are illuminated by the solar H Lyman $\alpha$ flux. Therefore, temporal and spatial variations of the solar irradiance are reflected in the interplanetary background. Quémerais and Bertaux (2002) have shown that the SWAN-SOHO maps of the interplanetary background emission can be used on a routine basis to produce maps of the relative variation of the solar illuminating flux at Lyman $\alpha$. In this study, we will improve the method developed by Quémerais and Bertaux (2002).

The interplanetary background data have been modeled by various authors. The most complete calculations have been described by Quémerais and Izmodenov (2002) and Katushkina et al. (2015).

Following Quémerais (2002), we can express the interplanetary background intensity at frequency $v$ as a line of sight integration of the local emissivity toward the observer multiplied by the extinction probability between the scattering point and the observer.

$$
I(\vec{r}, \vec{\Omega}, v)=\int_{0}^{\infty} \varepsilon(\vec{r}+s \vec{\Omega},-\vec{\Omega}, v) e^{-\tau_{v}(\vec{r}+s \vec{\Omega}, \vec{r})} \mathrm{d} s
$$

where $\vec{r}$ is the position of the observer, and $\vec{\Omega}$ the direction of the line of sight. The optical thickness between the scattering point and the observer is expressed by $\tau_{v}(\vec{r}+s \vec{\Omega}, \vec{r})$. The local emissivity at each point can be split between the single scattering term and the multiple scattering, $\varepsilon=\varepsilon_{0}+\varepsilon_{m}$.

The single scattering emissivity is proportional to the product of solar flux at frequency $v$, the extinction between the Sun and the scattering point, the H Lyman $\alpha$ scattering cross section, and the local scattering profile at frequency $v$. After integration over frequency, it can be simplified as the product of the solar flux multiplied by the primary intensity term, including the extinction between the Sun and the first scattering point. This extinction is negligible within a few astronomical units from the Sun.

The multiple scattering term of the emissivity at each scattering point is obtained by an integration over the whole sky, excluding the Sun, of the interplanetary emissivity in the direction of that scattering point.

This means that the photons contributing to the single scattering term come from the same point on the Sun directly aligned with the scattering point. The main emission actually comes from beyond the orbit of Mars as emissivity peaks somewhere around 2 to 2 AU. On the other hand, the multiple scattering term originates from a much wider region on the Sun (see Figure 1). As shown by Quémerais et al. (1996), the 


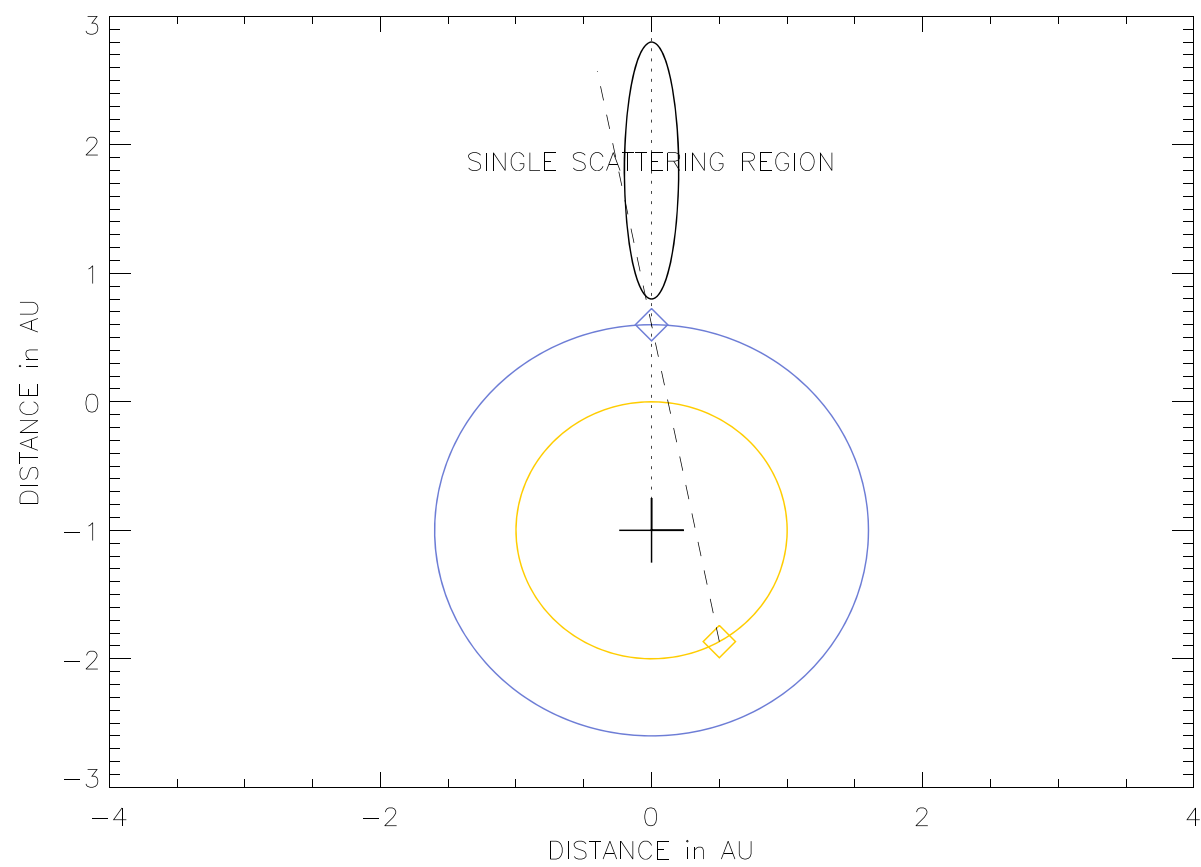

Figure 1. Scheme showing the geometry of observation. The Sun is marked by a cross, Mars by a blue diamond, and Earth (Solar Heliospheric Observatory) by an orange diamond. The solar photons toward Mars are mainly scattered behind Mars as emissivity is largest around 2-3 AU from the Sun. This region where emissivity is largest is shown as the main source of single scattering photons. The source region for multiple scattering photons is not shown as it extends to everywhere in the interplanetary medium. Note that for this geometry the two planets are illuminated by opposite sides of the Sun. In case of a strong modulation of the solar flux, this would be a case when the Solar Wind ANisotropies method would be most effective.

multiple scattering term is not directly proportional to the solar flux emitted by the Sun in the direction of the scattering point. In fact, multiple scattered photons can come from any point of the surface of the Sun, although most come from the hemisphere of the Sun facing that direction. The distribution of the source of the photons on the Sun depends on the actual geometry of the observation; however, a good approximation is obtained by using an average of the solar flux integrated over the disk of the Sun, noted $\langle F\rangle$.

Equation (1) can be simplified as follows

$$
I(t)=F(t) J_{p}+\langle F\rangle J_{S} .
$$

As discussed in Quémerais et al. (2008), the interplanetary medium is optically thin within a few astronomical units from the Sun. However, it is necessary to include the multiple scattering term because the observed sky brightness is integrated over tens of astronomical units and extends beyond the inner heliosphere where the optically thin approximation is applicable.

The solar flux value relevant for the multiple scattering term can be approximated by an average of the solar irradiance measured from the Earth position over a chosen period of time, typically one or two solar rotations. We can express the temporal variations of the interplanetary intensity measured in a given direction $I(t)$ as

$$
I(t)=F(t) J_{p}+\langle F\rangle J_{s}
$$

The directly illuminating solar flux is given by $F(t)$, and its time average is noted by $\langle F\rangle$. The "primary" brightness is given by $J_{p} . J_{s}$ is the "secondary" term due to multiple scattering. Both $J_{p}$ and $J_{s}$ correspond to the integral along the line of sight of the local emissivity for unit flux and multiplied by the extinction between the scattering point and the observer. The emissivity for the single scattering term also takes into account the Lyman $\alpha$ scattering phase function (cf. Quémerais et al., 2008). The time average of the brightness in a 
given direction is given by

$$
\begin{aligned}
\langle I\rangle & =\left\langle\left(F(t) J_{p}+\langle F\rangle J_{s}\right)\right\rangle \\
& =\langle F\rangle\left(J_{p}+J_{s}\right) .
\end{aligned}
$$

The modulation of the SWAN data can therefore be expressed as a function of the modulation of the solar flux:

$$
\begin{aligned}
\frac{I(t)-\langle I\rangle}{\langle I\rangle} & =\frac{F(t) J_{p}-\langle F\rangle J_{p}}{\langle F\rangle\left(J_{p}+J_{s}\right)} \\
& =\frac{F(t)-\langle F\rangle}{\langle F\rangle}\left(\frac{J_{p}}{J_{p}+J_{s}}\right) .
\end{aligned}
$$

If we define the damping factor, $D=\frac{J_{p}}{J_{p}+J_{s}}$, we see that the amplitude of the modulation of the solar flux is attenuated in the interplanetary background in proportion to $D$. For true optically thin media, $D$ is equal to 1 , and the amplitude of the modulations of the solar flux is preserved. As the optical thickness increases, this factor $D$ approaches 0 and the modulations disappear.

\subsection{Deriving the Solar Flux From Modulations}

The results obtained above can be used to derive the solar flux from the modulations of the SWAN data. We can define the SWAN index, $S(\theta)$, as the modulation of the SWAN measurements in a given direction. For example, in ecliptic coordinates where $\theta$ is the ecliptic longitude,

$$
S(\theta)=\frac{I(\theta)-\langle I\rangle}{\langle I\rangle}=\frac{F(\theta)-\langle F\rangle}{\langle F\rangle} D(\theta) .
$$

We can then express the solar flux in direction $\theta$ as

$$
F(\theta)=\langle F\rangle \times\left(1+\frac{S(\theta)}{D(\theta)}\right) .
$$

$\langle F\rangle$ in equation (7) technically applies to the average obtained in a given direction. Because of the solar rotation, we can assume that the flux average $\langle F\rangle$ is the same in every direction. This assumption is reasonable if the average is made over at least two solar rotations.

As presented in Quémerais \& Izmodenov, 2002 (2002, Table 5), the value of $D(\theta)$ can be modeled. The values in the sixth column (labeled $I_{0} / I_{n}$ ) give the ratio between primary and total brightness for radial lines of sight originating at $1 \mathrm{AU}$. As shown in that paper, it is expected that lines of sight through the downwind cavity will have smaller values of $D$ (i.e., a larger damping effect) than lines of sight in the upwind direction.

A simpler approach is to assume that $D$ is independent of direction. Therefore, we can determine the value of $D$ by a simple linear regression between the SWAN data modulations and the EUVM flux modulations. We can then apply this value to predict the flux in any direction and verify that the values derived for Mars agree with the EUVM measurements.

\section{Application to the EUVM and SWAN Data Sets}

\subsection{EUVM Data Set}

MAVEN's primary mission is to understand how Mars' atmosphere has evolved over time, and it does this with a suite of instruments designed to measure solar forcing at Mars and how the Martian atmosphere responds. EUVM-MAVEN measures calibrated solar irradiance in three EUV wavelength bands using filter photometers in order to characterize the EUV input to the Mars atmosphere. Measurements are made at 1-s cadence for most periods when the spacecraft is not in eclipse, approximately $63 \%$ of the time. Of relevance for this study is the EUVM Lyman $\alpha$ band. Ninety percent of the measured signal in this band originates between 121 and $122 \mathrm{~nm}$ (Thiemann, 2016). This band has been cross-calibrated with measurements from the Solar-Stellar Irradiance Comparison Experiment (McClintock et al., 2005) on board the Solar Radiation and Climate Experiment (Rottman, 2005) with 2.1\% agreement between the instruments (Thiemann et al., 2017). The EUVM data set used in this work covers a period from January 2014 to August 2018. 


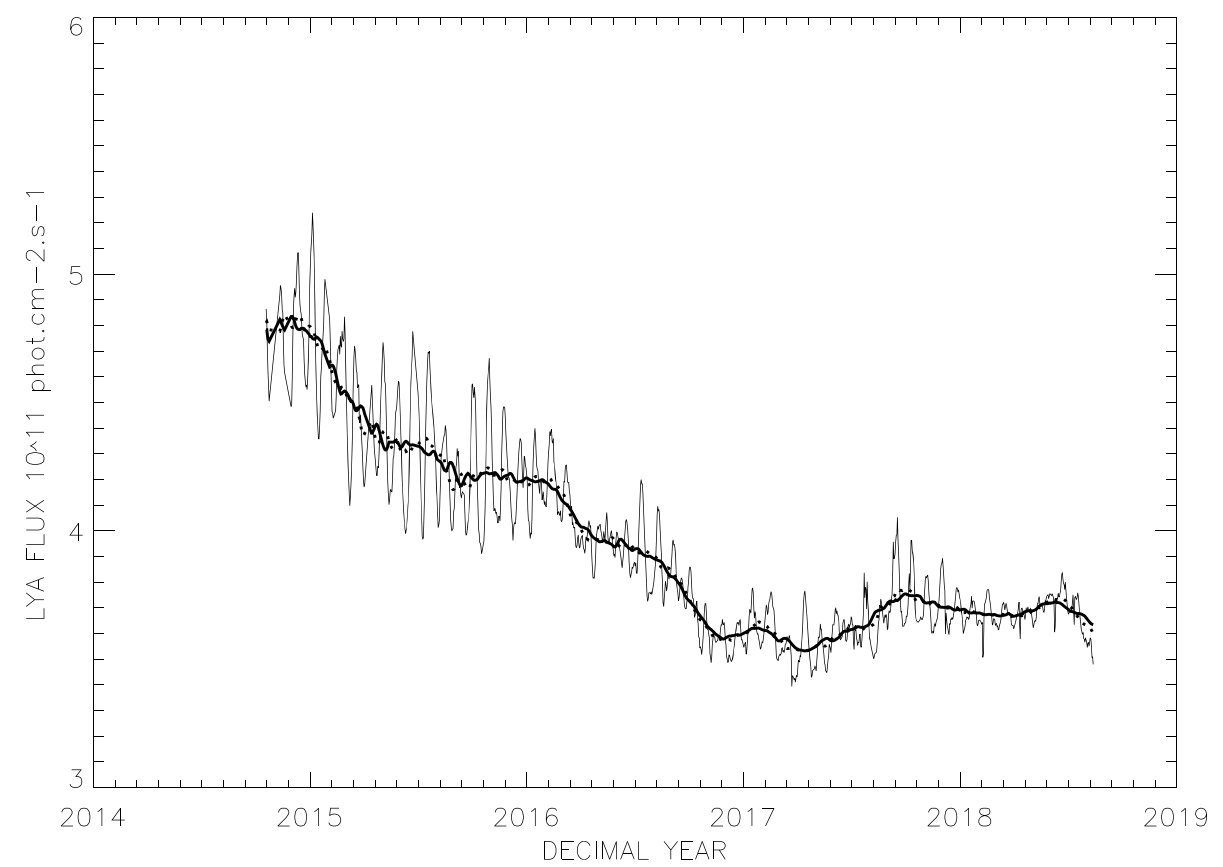

Figure 2. Extreme Ultraviolet Monitor Lyman $\alpha$ flux measurements obtained between 2014 and 2018 at Mars. The solar flux is scaled to the value at $1 \mathrm{AU}$. Rotational modulations are clearly seen. The dotted line shows the smoothed value obtained with a gliding average over two solar rotations. The solid line shows the fit with a Chebishev function over the same period. The Chebishev fit is less affected by boundary values but gives a similar result for the overall range.

\subsection{SWAN Data Set}

The SOHO mission is an international cooperation between ESA and NASA, dedicated to the study of the Sun and the heliosphere. The spacecraft is at the Lagrange point L1 and has an unobstructed view of the Sun and the inner heliosphere. The mission was launched in December 1995 and has been monitoring the Sun continuously since the beginning of the nominal mission in the Spring of 1996, except for a short period from June to November 1998. SWAN-SOHO is dedicated to the study of the interplanetary Lyman $\alpha$ glow (Bertaux et al., 1995). It is composed of two identical units placed on opposite sides of the spacecraft, and each one measures the interplanetary UV emission over half of the sky on a daily basis.

The SWAN data set consists of maps of the interplanetary background obtained by covering the whole sky in roughly $24 \mathrm{hr}$. Each unit of SWAN is equipped with a two-axis scanning system and a periscope that allows it to point its 5 by $5^{\circ}$ field of view in any direction of the hemisphere visible to that unit. The instantaneous field of view of each unit is divided into a 5 by 5 pixel array. Each pixel has a 1 by $1^{\circ}$ field of view, and a sufficient signal-to-noise ratio is obtained by accumulating counts for $13 \mathrm{~s}$. Taking into account mechanism movements and processing times, individual measurements are performed every $15 \mathrm{~s}$, thus taking roughly $24 \mathrm{hr}$ to complete the map. Data from both units are combined, corrected for pixel sensitivity and nonuniformity, and then combined in a full sky map of the Lyman $\alpha$ emission.

Modulations of the interplanetary background have been described by various authors (Pryor et al., 1992; Quémerais et al., 1996) and linked to the presence of active regions on the solar surface. These active regions are brighter than the quiet Sun at Lyman $\alpha$ and produce a local increase of the solar Lyman $\alpha$ flux. When the Sun rotates, the variation of the solar flux is seen as a modulation of the interplanetary background.

Early in the SOHO mission, Bertaux et al. (2000) identified the possibility of deducing the presence of active regions on the far side of the Sun-hidden from observers at Earth or L1 - by mapping the rotational modulations of the interplanetary background. The existence of a local increase of the solar flux in the region of space that is illuminated by the far side of the Sun shows that there is an active region of that side of the Sun. Quémerais and Bertaux (2002) used this idea to create an algorithm that could be used to predict the solar flux that would reach Earth up to half a solar rotation in advance. 


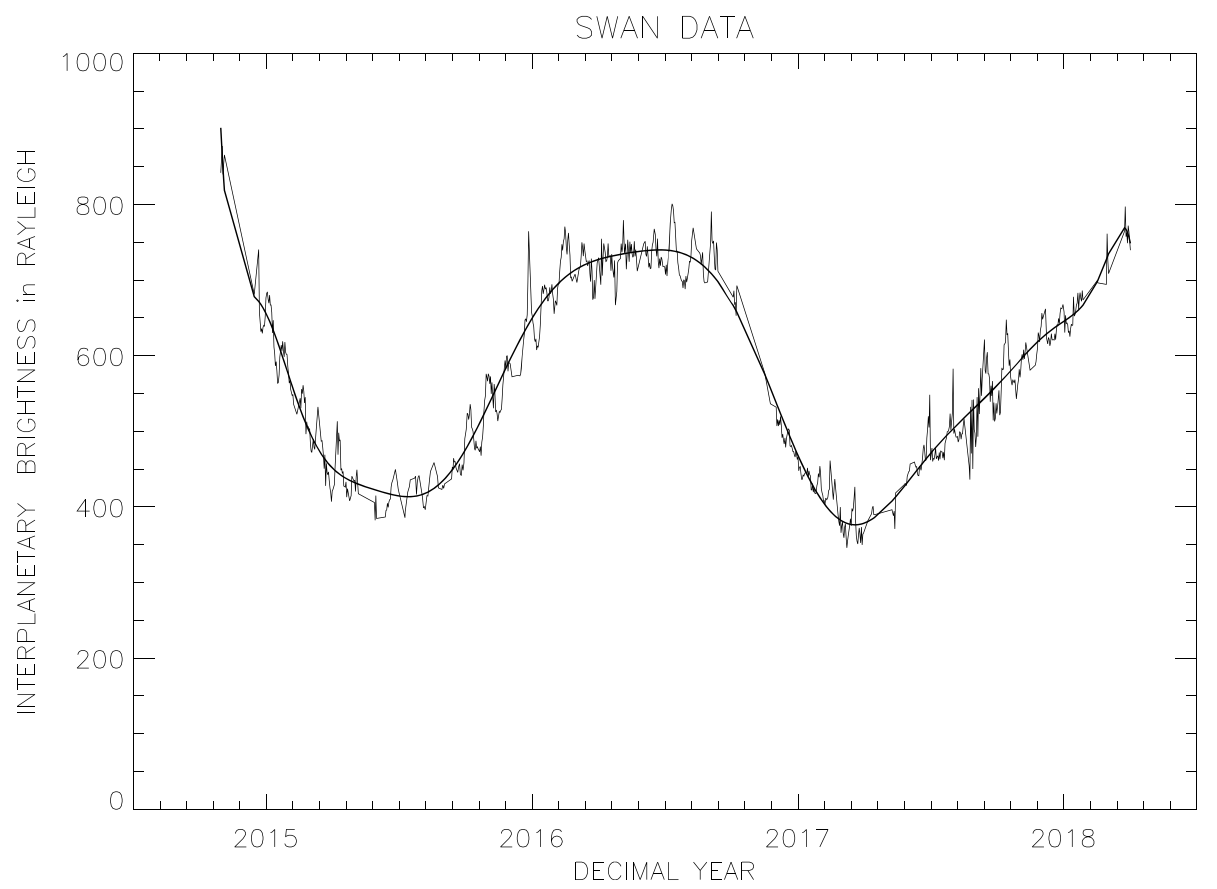

Figure 3. SWAN interplanetary Lyman $\alpha$ brightness measurements obtained between 2014 and 2018 in the direction of Mars. The solid line shows the result of the Chebishev fit. It is used to remove the temporal variations created by the orbit of Solar Heliospheric Observatory around the Sun. The brightness is maximum when the line of sight is close to the upwind direction and minimum when the line of sight is close to the downwind direction. SWAN = Solar Wind ANisotropies.

Starting in 2007, full sky observations are performed on a daily basis except for a few campaigns dedicated to bright comets. Most maps are complete except for two masked regions. One is due to the presence of two Sun shields that protect the SWAN units from direct sunlight. The second one is caused by obstruction by the spacecraft mechanical structure in the antisun direction.

For the present study we extracted the value obtained in the direction of Mars as seen from SOHO from the daily SWAN maps. Figure 3 shows the SWAN data in Rayleigh as a function of time. The 1-year modulation is caused by the orbit of SOHO around the Sun. The modulations of the interplanetary brightness due to the solar rotation are clearly visible in the data with a period of 27 days.

\subsection{Deriving the Damping Factor}

We use the data shown in Figures 2 and 3 to derive the damping factor, $D$, introduced in the previous section. The SWAN data modulations are given by $S$. From equation (6), we have

$$
S=\left(\frac{I(\theta)-\langle I\rangle}{\langle I\rangle}\right)=D\left(\frac{F(\theta)-\langle F\rangle}{\langle F\rangle}\right) .
$$

$D$ can be determined by performing a simple linear regression between the modulations of the two data sets.

Figure 4 shows a superposition of the modulations for data sets from 2015 to the end of 2016. This time period is the most interesting for our study as there are clear 27-day modulations due to solar activity in the declining phase of the solar cycle. Comparatively, 2017 has less activity, and therefore the modulations have smaller amplitude. The correlation between the two data sets is excellent as expected from previous studies. The black solid line in Figure 4 shows the modulation of the SWAN background measurements, $S$. The green solid line corresponds to the solar flux modulations seen by EUVM. The data in Figure 4 are shown at their native time resolution. The gaps in the SWAN data are due to the obstructions described above. Because of this, we have resampled the EUVM data to the SWAN observation times. Based on the previous description and the data shown in Figure 5, we find that the damping factor $D$ is equal to $0.903 \pm 0.036$.

Before discussing this result, it is necessary to consider that the hydrogen atoms in the interplanetary medium are not illuminated by the whole solar line but only by its central part. This is due to the relatively 


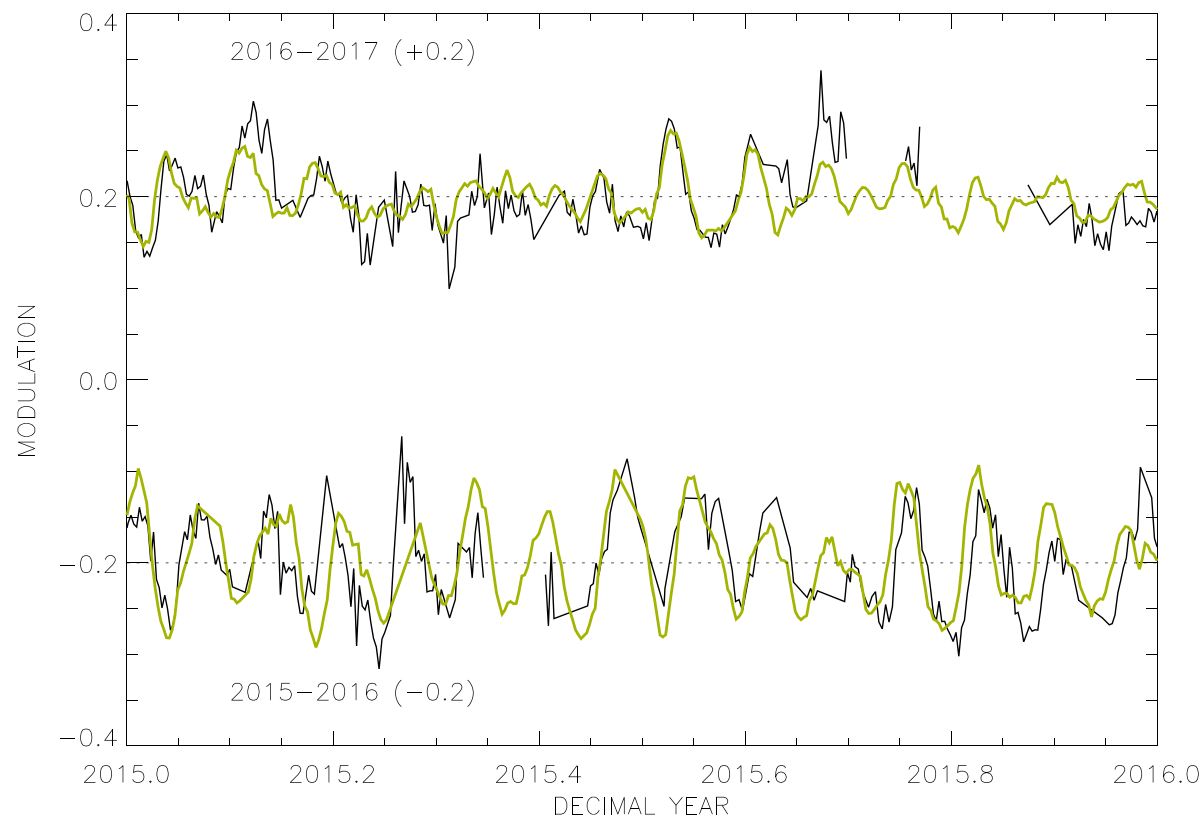

Figure 4. Modulations of the EUVM (green solid line) and SWAN data (black solid line) as a function of time in 2015 and 2016. For clarity, data for 2015 are shifted by -0.2 and data for 2016 are shifted by 0.2 . During the period displayed, large modulations are observed due to the solar activity in the declining phase of the solar cycle. The correlations of the modulations in the two data sets are excellent. The SWAN data show more data gaps than the EUVM data due to the obstructions described in the text. EUVM = Extreme Ultraviolet Monitor; SWAN = Solar Wind ANisotropies.

small velocity of the $\mathrm{H}$ atoms in the interplanetary medium in the solar rest frame. Hydrogen atoms have a bulk velocity of $\pm 20-25 \mathrm{~km} / \mathrm{s}$, depending on whether they are upwind or downwind from the Sun, and a thermal velocity of less than $15 \mathrm{~km} / \mathrm{s}$. Conversely, the solar line has a width that corresponds to a Doppler shift of $150 \mathrm{~km} / \mathrm{s}$ in the solar rest frame. Early studies of the solar Lyman $\alpha$ line (Fontenla et al., 1988; Vidal-Madjar, 1975) have shown that this line has a different profile for different types of activity (e.g., active regions or quiet Sn for instance). Therefore, the line profile changes during the solar cycle. Various authors have tried to model the variations of the line profile with solar activity (Fontenla et al., 1988; Vidal-Madjar, 1975). The most recent models of the solar line profile are based on measurements from SUMER-SOHO (Emerich et al., 2005; Kretzschmar et al., 2018).

We have tested the importance of the relation between the line center flux and the total flux in our study by doing a linear regression between the total flux modulations and the line center flux modulations. Two relations have been examined, Emerich et al. (2005) and Kretzschmar et al. (2018). The model by Emerich et al. (2005) gives the following relation where $F$ is the solar flux in units of per square centimeter per second and $F_{\text {core }}$ is the line center flux in units of per square centimeter per second per Angstrom.

$$
F_{\text {core }}=0.64 \times F^{1.21}(\text { Emerich }) \text {. }
$$

The model by Kretzschmar et al. (2018) represents the solar Lyman $\alpha$ flux profile at a given wavelength $F(\lambda)$ by a linear relation with the total flux. For instance, in the case of the line center, $\lambda=121.566 \mathrm{~nm}$,

$$
F_{\text {core }}=-1.61+1.112 \times F(\text { Kretzschmar }) \text {. }
$$

The two models give a slightly different result for the value of $F_{\text {core }}$, but they are within 5\% of each other. Another model by Kowalska-Leszczynska et al. (2018) was also published recently. It gives results similar to Kretzschmar et al. (2018) at line center and is not used in this work.

We have applied the two model results to the EUVM data set and derived the modulation proportionality coefficient between the line center flux and the total line flux. We find that the proportionality coefficient between the line center flux and the total line flux is equal to 1.2 for the Emerich et al. (2005) model and 1.34 for the Kretzschmar et al. (2018) model. It means that if the total line flux has a modulation of amplitude 


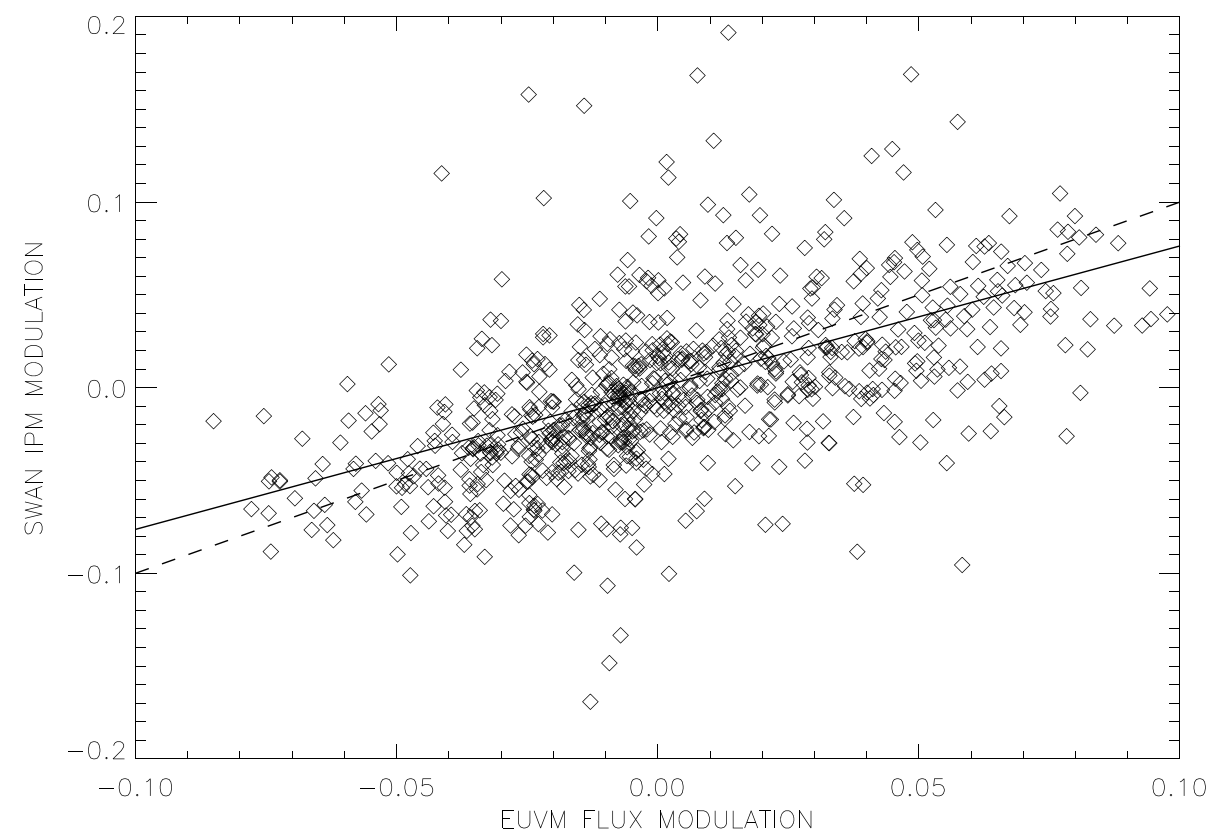

Figure 5. Linear regression between the two data sets shown in the previous figure. These data include all available measurements obtained early 2014 to mid-2018. The dashed line shows the optically thin case $(D=1)$. This is the upper limit for the value of $D$. The solid line shows the result found for this analysis.

A, then the line center flux shows a modulation with amplitude 1.2 times A in the case of the Emerich et al. (2005) model. The line center flux shows a modulation with amplitude 1.34 times $\mathrm{A}$ in the case of the Kretzschmar et al. (2018) model.

Since the bulk velocity of the $\mathrm{H}$ atoms in the solar rest frame varies between roughly $+20 \mathrm{~km} / \mathrm{s}$ (downwind, red shifted population) and $-25 \mathrm{~km} / \mathrm{s}$ (upwind, blue shifted population), we have computed the average of the modulation proportionality coefficient in that velocity range. We find a mean value of $1.32 \pm 0.1$.

This shows that the modulations of the solar Lyman $\alpha$ illuminating flux are larger at line center by a factor of 1.2-1.3. This is not surprising given that the line center is formed at higher and more variable regions of the Sun's atmosphere than the line wings (Vernazza et al., 1973). This value of the damping factor is biased because we have derived it based on the modulations between the SWAN data and the total line flux measured by EUVM. The true value of the damping factor must be derived from the SWAN modulations and line center flux. We can compare the two modeled line center flux values. In the case of the Emerich et al. (2005) model, we find that the damping factor $D$ is equal to $0.745 \pm 0.030$. Using the Kretzschmar et al. (2018) model, we find that the damping factor is equal to $0.678 \pm 0.027$. Both models therefore give similar estimates of the damping factor.

This result is an independent confirmation that multiple scattering is a significant process in the interplanetary medium. In the optically thin case, the damping factor, which is the ratio between the single scattering intensity to the total intensity, must be equal to 1 . However, we find that the interplanetary background modulations are damped by a factor of $\sim 0.7$. This means that on average for an observer at 1 AU, the single scattering term only accounts for $70 \%$ of the total intensity.

Finally, we will point out that the empirical result found here is in complete agreement with the results of Quémerais and Izmodenov (2002) as given in the sixth column of Table 5 of that work that estimates the ratio between the single scattering term and the total intensity. This confirms the numerical approach to compute radiative transfer in the interplanetary medium presented by these authors.

\section{Deriving the Solar Flux From SWAN Data}

Now that we have determined the damping factor, we can apply equation (5) to estimate the solar flux toward Mars based on the combination of Earth-based measurements and the SWAN index of interplanetary modulations. The result will be compared to the actual values measured by EUVM on MAVEN. 


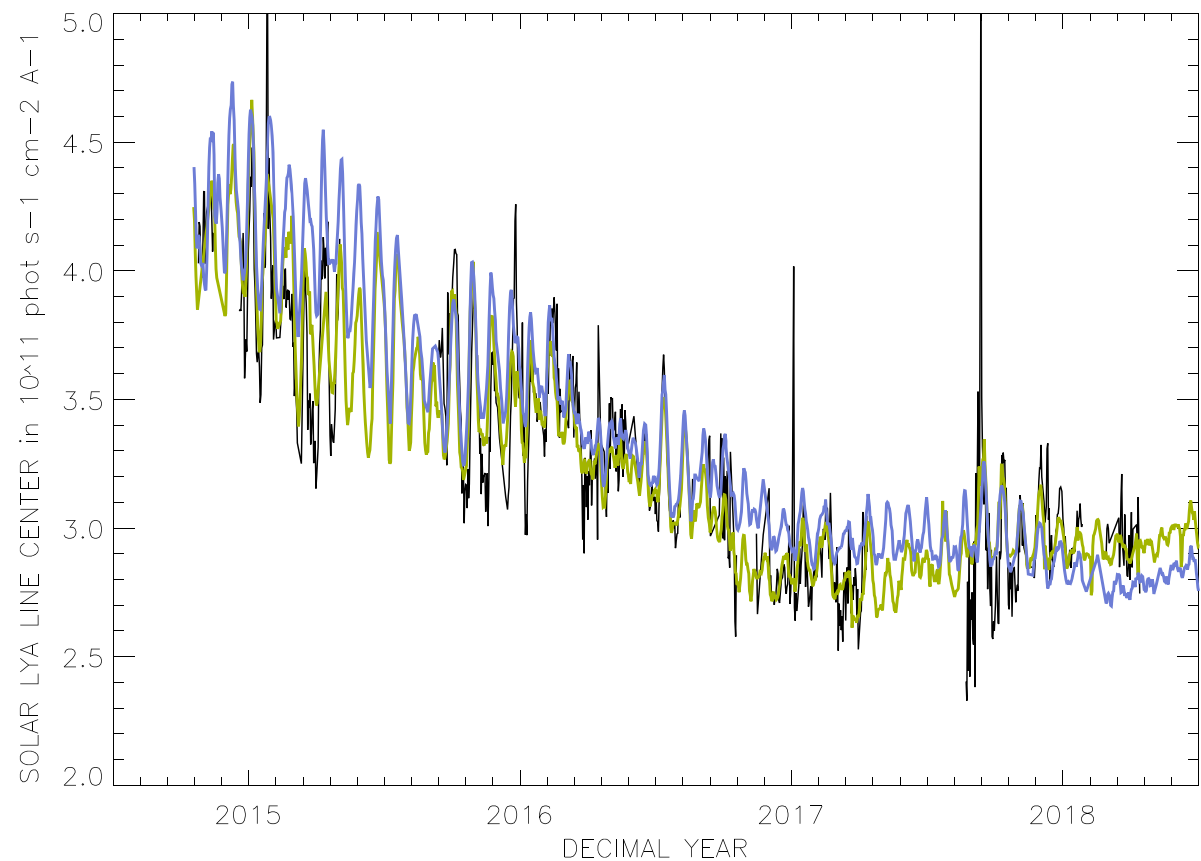

Figure 6. Comparison between the solar line center illuminating flux at Lyman $\alpha$ measured by Extreme Ultraviolet Monitor on Mars Atmosphere and Volatile EvolutioN (green solid line) and the SWAN model based on equation (5) and a damping factor of 0.68 , as described in the text. The SWAN-derived flux is shown by the black line. Note that the SWAN data have more gaps and are generally more noisy. The blue solid line shows the result of the time-shift computation which is systematically higher at the beginning of the data set, while the SWAN model agrees better. SWAN $=$ Solar Wind ANisotropies.

Equation (7) is applied directly to derive the solar line center Lyman $\alpha$ flux. In that case, we must apply the correct damping factor found by the linear regression, that is, $D=0.68$.

$$
F=\langle F\rangle \times\left(1+\frac{S(\theta)}{0.68}\right)
$$

Since the values measured by EUVM correspond to the total line flux, we have converted these values using the relationship of Kretzschmar et al. (2018).

Figure 6 shows the result obtained by applying equation (5) with $D=0.68$. The mean solar Lyman $\alpha$

Table 1

Accuracy of the Predicted Flux Toward Mars

\begin{tabular}{lcc}
\hline & \multicolumn{2}{c}{ Fraction of values } \\
\cline { 2 - 3 } Accuracy & SWAN method & Position-shifted method \\
\hline 0.01 & 0.19 & 0.12 \\
0.02 & 0.35 & 0.25 \\
0.03 & 0.47 & 0.45 \\
0.04 & 0.59 & 0.63 \\
0.05 & 0.67 & 0.74 \\
0.06 & 0.76 & 0.80 \\
0.07 & 0.83 & 0.87 \\
0.08 & 0.87 & 0.92 \\
0.09 & 0.90 & 0.95 \\
0.10 & 0.93 & 0.96 \\
\hline
\end{tabular}

Note. SWAN = Solar Wind ANisotropies irradiance is obtained by applying a 54-day gliding average to the Earth-based data as provided by the Lyman $\alpha$ composite from the LASP LISIRD web page (see Woods et al., 2000). Note that the LISIRD data for the total line flux have been transformed to line center flux values.

As shown in Figure 6, the predicted flux ( black line in the figure) is more noisy than the observations from EUVM (solid blue line). However, when strong modulations are present, as in early 2015, the result of our empirical model tracks the actual flux very well. Finally, we have estimated the accuracy of the model by computing the relative difference between the predicted fluxes from SWAN and the time-shifting method to the actual flux measured by EUVM. The comparison to the two methods is shown in Table 1 .

We see that the results of both methods give estimates that are almost always within $10 \%$ of the actual value measured by EUVM. The SWAN-based method produces a larger fraction of the most highly accurate estimates, that is, within $2-3 \%$ of the actual values, but also has a larger number of the least accurate results. On average, both methods give similar results. 
Because of the larger noise of the SWAN data and some stellar contamination that is difficult to remove routinely, the SWAN estimates have a few outliers that are not present in the results of the time-shifted flux estimate. In particular when there are little or no modulations in the data, the noise of the SWAN data increases the inaccuracy of the estimate.

On the other hand, when there are strong modulations, like in early 2015, the SWAN-based results are better, because the time-shifted flux method neglects the temporal variations of the solar surface magnetism that creates the modulations. When the Sun is more active, the SWAN results are more accurate.

Finally, another source of error in the SWAN-based estimates is the parallax effect caused by the scattering of the solar photons in the interplanetary medium. The peak in the scatter of the solar photons comes from atoms that are at a distance of about 2 to $3 \mathrm{AU}$ from the Sun, depending on the position relative to the upwind direction. This creates a small angle shift for flux estimates that are perpendicular to the Sun-Earth line. Quémerais et al. (1996) studied the impact of this effect and showed that it could be corrected using a model. That is beyond the scope of this work but can explain some of the inaccuracies in the SWAN-based method.

\section{Conclusion and Discussion}

In this paper, we have presented a new approach to derive the Lyman $\alpha$ solar spectral irradiance in every direction based on a combination of the Lyman alpha composite (Woods et al., 2000) data and the SWAN interplanetary background measurements. The Lyman alpha composite data provide the mean value of the solar flux, while the SWAN data modulations give the temporal variations around the mean value. This method is a generalization of the method presented by Quémerais and Bertaux (2002) used to forecast the solar flux toward Earth half a solar rotation ahead in time.

Our new approach shows that there is a linear relation between the modulations of the solar Lyman $\alpha$ flux and the modulations of the interplanetary background data. The main source for the modulations is the solar rotation. The proportionality coefficient between the solar modulations and the interplanetary data modulations is called the damping factor $D$. This factor is equal to the fraction of the single scattering term in the total interplanetary brightness.

In the case of an optically thin medium, the damping factor $D$ is equal to 1 ; this means that the modulation at the Sun and in the interplanetary medium have the same amplitude. At large distance from the Sun, $D$ tends toward 0, and all modulations are damped. This effect was seen by Quémerais et al. (1996) when analyzing modulations of interplanetary data obtained by the Ultraviolet Spectrograph on Voyager 2.

In the case of the SWAN-SOHO data, we see that the damping factor between interplanetary data modulations and modulations in the solar irradiance is equal to 0.9. However, a correction must be introduced to account for the fact that the $\mathrm{H}$ atoms in the interplanetary medium are only illuminated by the photons at line center. If this is taken into account, by using the Kretzschmar et al. (2018) model of the solar Lyman $\alpha$ line shape, we find a damping factor close to 0.68 . This is obtained by neglecting spatial variations of $D$, which will be discussed below.

This study shows that at $1 \mathrm{AU}$ from the Sun, single scattering only accounts for roughly two thirds of the total brightness. At that distance, the hydrogen number density is very small, and one would expect that the interplanetary medium is optically thin. However, we must not forget that the scattering of solar photons by hydrogen atoms happens everywhere in the heliosphere and that therefore the contribution from multiple scattering at larger distance from the Sun represents one third of the total intensity. This is in agreement with the computations of Quémerais and Izmodenov (2002).

It should be noted that detailed models of the background intensity show that the damping factor varies with the observation geometry (Quémerais \& Izmodenov, 2002). Our empirical determination of the value of $D$ by comparison of the data from EUVM and SWAN only gives an average value relevant for these data sets.

In the last section of this work, we have applied the new algorithm to compute the solar illuminating flux in the direction of Mars. We have also computed the flux in the case of the time-shifting flux method which is most often used. The results have been compared to the actual flux measured by EUVM. We find that in general both methods give similar results. The SWAN method is a bit more accurate when there are strong modulations of the solar flux. On the other hand, in period of low modulation, the SWAN results are not as good because of the larger noise in the SWAN background data. 
Finally, it should be mentioned that because the peak emissivity is around 2 to $3 \mathrm{AU}$ in the inner heliosphere, there is a small deviation angle for the SWAN method in the determination of the solar flux in the direction perpendicular to the Sun-Earth line. This effect can be removed with a model-dependent correction (Quémerais et al., 1996).

In the future, this study will be expanded to try and derive more accurate damping factors depending on the position of the observer. For instance, based on the computations from Quémerais and Izmodenov (2002), we expect that the damping factor changes when the observer moves from the upwind to the downwind region. An average value is expected when the observer is crosswind. We will study this effect when more data become available.

Finally, we have estimated the accuracy of the SWAN-based flux model to Mars by computing the relative difference between the predicted flux and the actual flux measured by EUVM. This is the first opportunity to validate the SWAN-based flux model with a solar spectral irradiance measurement at a planet.

Acknowledgments

SOHO is a mission of cooperation between ESA and NASA. SWAN was developed as a cooperation between France (CNRS and CNES) and Finland (Finnish Meteorological Institute). The authors acknowledge partial support from the International Space Science Institute (ISSI, Bern) by hosting the SHAPE team dedicated to the solar Lyman $\alpha$ line. M. S. was supported by NASA contract NAS5-97045 (SORCE) at the University of Colorado. E. T. was supported by LWS grant

NNX16AE86G. The SWAN data used in this analysis are available at the following link, on the website (https:// sohowww.nascom.nasa.gov/data/ archive/). A description of the data can be found at the website (http://swan. projet.latmos.ipsl.fr/). The SOLSTICE data can be found at the LISIRD website (http://lasp.colorado.edu/ lisird/data/lyman_alpha_model_ssi/). The MAVEN EUV SSI daily average is on LISIRD at the website (http://lasp. colorado.edu/lisird/data/mvn_euv_l3_ daily/). The EUVM data are also part of the MAVEN data sets and can be found at the website (https://pds.nasa.gov/).

\section{References}

Ajello, J., Stewart, A., Thomas, G., \& Graps, A. (1987). Solar cycle study of interplanetary Lyman-alpha variations-Pioneer Venus Orbiter sky background results. Astrophysical Journal, 317, 964-986.

Bertaux, J.-L., \& Blamont, J. (1971). Evidence for a source of extraterrestrial hydrogen Lyman-alpha emission. Astronomy \& Astrophysics, 11, 200-217.

Bertaux, J.-L., Kyrölä, E., Quémerais, E., Pellinen, R., Lallement, R., Schmidt, W., et al. (1995). SWAN: A study of solar wind anisotropies on SOHO with Lyman alpha sky mapping. Solar Physics, 162, 403-439.

Bertaux, J.-L., Quémerais, E., Lallement, R., \& Lamassoure, E. (2000). Monitoring solar activity on the far side of the Sun from sky reflected Lyman $\alpha$ radiation. Geophysical Research Letters, 27, 1331-1334.

Emerich, C., Lemaire, P., Vial, J.-C., Curdt, W., Schüle, U., \& Wilhelm, K. (2005). A new relation between the central spectral solar H I Lyman $\alpha$ irradiance and the line irradiance measured by SUMER/SOHO during the cycle 23. Icarus, 178, 429-433.

Eparvier, F., Chamberlin, P., Woods, T., \& Thiemann, E. (2015). The solar extreme ultraviolet monitor for MAVEN. Space Science Reviews, 195, 293-301.

Fontenla, J., Reichmann, E. J., \& Tandberg-Hanssen, E. (1988). The Lyman-alpha line in various solar features. I. Observations. Astrophysical Journal, 329, 464-481.

Jakosky, B. M., Lin, R. P., Grebowksy, J. M., Luhmann, J. G., Mitchell, D. F., Beutelschies, G., et al. (2015). The Mars Atmosphere and Volatile EvolutioN (MAVEN) mission. Space Science Reviews, 195(1-4), 3-48.

Katushkina, O., Izmodenov, V., \& Alexashov, D. (2015). Direction of interstellar hydrogen flow in the heliosphere: Theoretical modelling and comparison with SOHO/SWAN data. Monthly Notices of the Royal Astronomical Society, 446, 2929-2943.

Kowalska-Leszczynska, I., Bzowski, M., Sokół, J., \& Kubiak, M. (2018). Evolution of the solar Lyman $\alpha$ profile during the solar cycle. Astrophysical Journal, 852, 115.

Kretzschmar, M., Snow, M., \& Curdt, W. (2018). An empirical model of the variation of the solar Lyman- $\alpha$ spectral irradiance. Geophysical Research Letters, 45, 2138-2144. https://doi.org/10.1002/2017GL076318

McClintock, W. E., Rottman, G. J., \& Woods, T. N. (2005). Solar Stellar Irradiance Comparison Experiment II (SOLSTICE II): Instrument concept and design. Solar Physics, 230(1-2), 225-258.

Pryor, W. R., Ajello, J. M., Barth, C. A., Hord, C. W., Stewart, A. I. F., Simmons, K. E., et al. (1992). Solar Lyman-alpha latitude variation at solar maximum from interplanetary Lyman-alpha observations. Astrophysical Journal, 394, 363-377.

Quémerais, E. (2002). Angel dependent partial frequency redistribution in the interplanetary medium at Lyman $\alpha$. Astronomy and Astrophysiscs, 358, 353.

Quémerais, E., \& Bertaux, J.-L. (2002). 14-day forecast of solar indices using interplanetary Lyman $\alpha$ background data. Geophysical Research Letters, 29(2), 1018. https://doi.org/10.1029/2001GL013920

Quémerais, E., Bertaux, J. L., Lallement, R., Berthé, M., Kyrölä, E., \& Schmidt, W. (1999). Interplanetary Lyman $\alpha$ line profiles derived from SWAN/SOHO H Cell measurements: 1. The full sky velocity field. Journal of Geophysical Research, 104, 12,585-12,603.

Quémerais, E., \& Izmodenov, V. (2002). Effects of the heliospheric interface on the interplanetary Lyman $\alpha$ glow seen at 1 AU from the Sun. Astronomy \& Astrophysics, 396, 269-281.

Quémerais, E., Lallement, R., Sandel, B., \& Clarke, J. (2008). Interplanetary Lyman $\alpha$ observations: Intensities from Voyagers and line profiles from HST/STIS. In J. L. Linsky, V. V. Izmodenov, E. Möbius, \& R. von Steiger (Eds.), From the outer heliosphere to the local bubble, Space Sciences Series of ISSI (Vol. 31, pp. 151-162). New York, NY: Springer.

Quémerais, E., Sandel, B., \& de Toma, G. (1996). 26 day modulation of the Voyager Ly $\alpha$ data: Estimating the interplanetary hydrogen density. Astrophysical Journal, 463, 349-358.

Quémerais, E., Sandel, B., Izmodenov, V., \& Gladstone, G. (2013). Thirty years of interplanetary background data: A global view. In E. Quémerais, M. Snow, \& R.-M. Bonnet (Eds.), Cross-calibration of far UV spectra of solar system objects and the heliosphere (pp. 163-188), ISSI Scientific Report 13. New York, NY: Springer.

Rottman, G. (2005). The SORCE mission. Solar Physics, 230, 7-25.

Thiemann, E. M. (2016). Multi-spectral sensor driven solar EUV irradiance models with improved spectro-temporal resolution for space weather applications at Earth and Mars. (Doctoral dissertation University of Colorado at Boulder).

Thiemann, E. M., Chamberlin, P. C., Eparvier, F. G., Templeman, B., Woods, T. N., Bougher, S. W., \& Jakosky, B. M. (2017). The MAVEN EUVM model of solar spectral irradiance variability at Mars: Algorithms and results. Journal of Geophysical Research: Space Physics, 122, 2748-2767. https://doi.org/10.1002/2016JA023512

Thomas, G., \& Krassa, R. (1971). OGO 5 measurements of the Lyman alpha sky background. Astronomy \& Astrophsics, 30, $218-233$.

Vernazza, J. E., Avrett, E. H., \& Loeser, R. (1973). Structure of the solar chromosphere. Basic computations and summary of the results. Astrophysical Journal, 184, 605-632. 
Vidal-Madjar, A. (1975). Evolution of the solar Lyman alpha flux during four consecutive years. Solar Physics, 40, 69-86.

Woods, T. N., Tobiska, W. K., Rottman, G. J., \& Worden, J. R. (2000). Improved solar Lyman alpha irradiance modeling from 1947 through 1999 based on UARS observations. Journal of Geophysical Research, 105, 27,195-27,215. 